http://dx.doi.org/10.32911/as.2017.v10.n2.166

Aporte Santiaguino. 10 (2), 2017: 233-244

ISSN 2070-836X

\title{
Tipificación de prototipos de sistemas de producción agroforestal en la provincia de Tambopata, Madre de Dios
}

Typification of prototypes of agroforestry production systems in the province of Tambopata, Madre de Dios

\author{
Joel Peña Valdeiglesias ${ }^{1}$ y Julio Cesar Alegre Orihuela ${ }^{2}$
}

\section{RESUMEN}

Este trabajo se realizó con el objetivo de tipificar Prototipos de Sistemas de Producción Agroforestal en la provincia de Tambopata, Madre de Dios - Perú. Se trabajó con una población de 150 productores, de la que se tomó una muestra de 50. Se encontró que los Sistemas de Producción Agroforestal están agrupados en cinco tipos diferenciados entre sí. El SAF tipo I tiene una edad promedio de 10 años y 5 ha destinadas a la agroforesteria, con cultivos principales de copoazu y cacao asociado hasta con 4 especies forestales. El SAF tipo II tiene una edad promedio de 11,3 años y 8,8 ha destinadas a la agroforesteria, con cultivo de copoazu asociado hasta con 5 especies forestales. El SAF tipo III tiene una edad promedio de 12,3 años y 13,6 h destinadas a la agroforesteria, con cultivo de copoazu y piña asociado hasta con 3 especies forestales. Los SAF tipo IV y V tienen una edad promedio de 5,3 y 7,5 años respectivamente con menos de 7,6 ha destinadas a la agroforesteria, con cultivo de cacao joven asociada a 2 especies forestales.

Palabras clave: sistemas agroforestales; cacao; copoazu; tipificación; análisis multivariado.

\footnotetext{
ABSTRACT

1 Universidad Nacional Amazónica de Madre de Dios. Madre de Dios, Perú.

2 Universidad Nacional Agraria La Molina. Lima, Perú.
}

This work was performed with the objective of typifying prototypes of agroforestry production systems in the province of Tambopata (Madre de Dios - Peru). We worked with a population of 150 farmers/producers, a sample of 50 was taken. It was found that the agroforestry production systems are grouped in five different types from each other. The SAF type I has an average age of 10 years and 5 has of them destined to the 
agroforestry, with crops of copazu and cocoa associated with up to 4 forest species. The SAF type II has an average age of 11,3 years and 8,8 ha of them destined to the agroforestry, with crops of copazu associated with up to 5 forest species. The SAF type III has an average age of 12,3 years and 13,6 has of them destined to the agroforestry, with crops of copazu and pineapple associated with up to 3 forest species. SAFs type IV and $v$ have an average age of 5,3 and 7,5 years, respectively with less than 7,6 has destined to the agroforestry, with young cocoa crop associated with up to 2 forest species.

Keywords: agroforestry systems; cocoa; copoazu; typification; multivariate analysis.

\section{INTRODUCCIÓN}

El departamento de Madre de Dios, localizado en la Amazonía sur del Perú, está poblado por agricultores en forma dispersa que practican la actividad agroforestal en forma individual y están poco organizados, no existiendo reportes oficiales sobre estas intervenciones. Según Cáritas de Puerto Maldonado (Por la tierra, 2015), en Madre de Dios se promueve la agroforesteria desde el año 2006 a las poblaciones pobres y apoyan a cerca de 300 familias de las provincias de Tambopata y Tahuamanu. En la actualidad, se estima que solo en la provincia de Tambopata existen unas 150 parcelas agroforestales que tienen más de 4 años de establecidas.

La Agroforesteria es una actividad que se desarrolla hace buen tiempo en Madre de Dios como un modelo alternativo al monocultivo, la misma que ha sido promovida por algunas ONG y algunos proyectos estatales y que han obtenido buenos resultados iniciales, sin embargo, hasta la actualidad poco se conoce de la diversidad de prototipos de Sistemas Agroforestales (SAF) establecidos.

La tipificación permite conocer la organización conceptual de la diversidad existente en la agricultura campesina con una lista de unidades de producción representativa y la población vinculada a los sistemas de producción, por lo que es posible identificar grupos de descriptores que generalmente cumplen un papel importante en los ejercicios de tipificación y clasificación. (Escobar y Berdegué, 1990).

En base a estos antecedentes en este estudio nos planteamos los siguientes objetivos: 1) Identificar los prototipos de producción agroforestal representativos de la provincia de Tambopata y 2) Caracterizar las unidades de producción agroforestal identificadas.

\section{MATERIALES Y MÉTODOS}

La investigación se realizó en la provincia de Tambopata del departamento de Madre de Dios, ubicado en la Amazonía sur del Perú (figura 1), donde existen dos estaciones bien definidas: la húmeda, que se presenta desde los meses de noviembre hasta abril, caracterizada por la presencia de fuertes precipitaciones y altas temperaturas, y la es- 
tación seca que se caracteriza por la ausencia de lluvias y temperaturas más bajas, que se presentan desde los meses de mayo hasta octubre. El rango de precipitación fluctúa entre 1.500 y $2.500 \mathrm{~mm}$ anuales. Los suelos son aluviales antiguos, generalmente arcillosos rojos con poca fertilidad y $\mathrm{pH}$ ácido.

La población de estudio está conformada por 150 agricultores que tienen sistemas agroforestales con más de cuatro años de instaladas en la provincia de Tambopata, por lo que la muestra estimada para el presente estudio es de 50 agricultores.

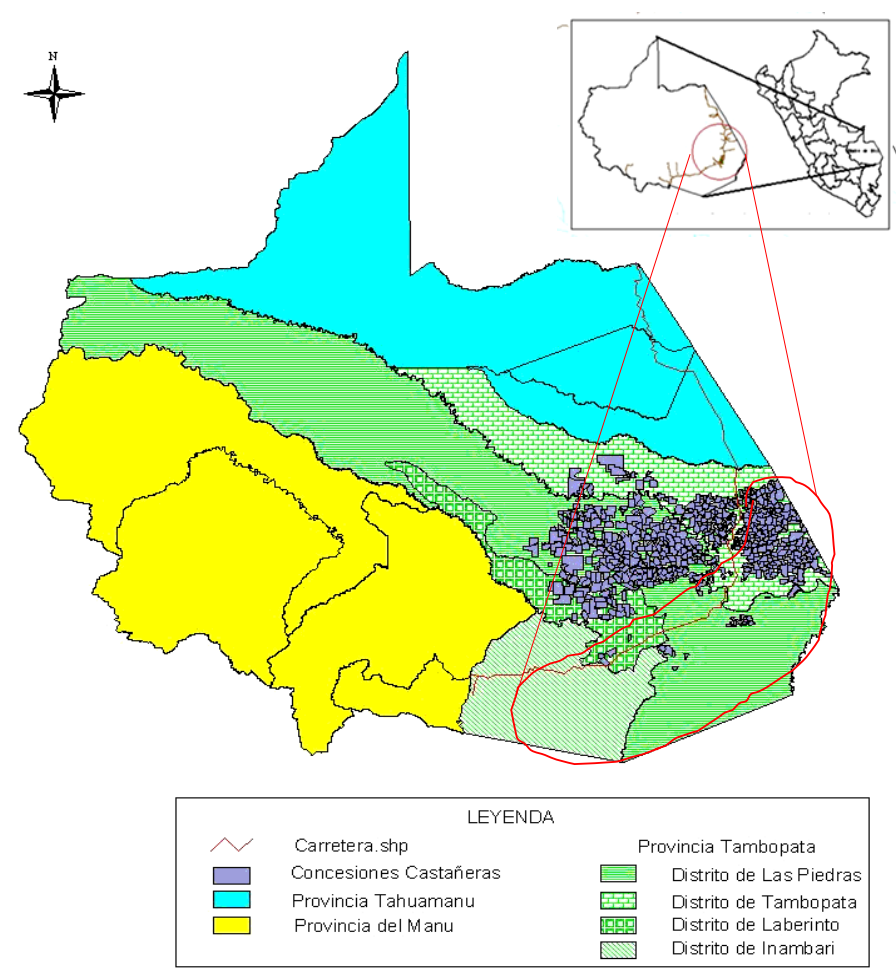

Figura 1. Localización del área de estudio

Para la tipificación de los Sistemas Agroforestales se aplicaron encuestas estructuradas a los agricultores, cuyas variables tienen los siguientes indicadores (Escobar y Berdegué, 1990): Tamaño de la finca, capitalización, estructura de la mano de obra disponible y empleada en la finca y fuera de ella, sistemas productivos existentes, nivel de intensificación tecnológica, tipo de tenencia de la tierra, calidad del suelo, composición del ingreso familiar, tipo y grado de articulación con los mercados de productos, localización geográfica y agroecológica, y capacidad de gestión, y de las metas y habilidades de los productores.

Para el procesamiento y análisis de la información se efectuaron análisis estadísticos multivariados planteados por Escobar y Berdegué (1990): Descarte de variables que 
carecen de poder discriminatorio, para lo cual se calculó los coeficientes de variación de cada una de las variables, y fueron descartadas las que tuvieron un coeficiente inferior a $50 \%$.

El Análisis de Componentes Principales se efectuó con la finalidad de reducir el número de variables, preferentemente a 2 o 3 componentes, de tal manera que pueda facilitar el análisis respectivo. Con estos datos se hizo la rotación de los ejes factoriales por el método de Varimax, donde se generaron las variables sintéticas para el análisis cluster o análisis de clasificación jerárquica produciéndose un dendrograma. Cada una de las ramificaciones mostradas en el dendrograma tiene el potencial de ser seleccionada como un tipo de sistema de finca. El nivel al cual se decida hacer la selección de tipos dependerá del balance que se haga de los siguientes elementos: a mayor cantidad de tipos mayor será la homogeneidad intratipos, mayor la heterogeneidad intertipos, y mayor el costo y el esfuerzo de investigación posterior.

\section{RESULTADOS}

De las 50 encuestas efectuadas, todas fueron válidas, y cada encuesta tuvo 64 variables evaluadas, de los cuales solo 15 variables se utilizaron para hacer la tipificación, teniendo en cuenta que su coeficiente de variabilidad fuera superior a $50 \%$.

\section{Análisis de Componentes Principales}

Haciendo el cálculo analítico de los factores a partir de las variables originales, y de acuerdo a la tabla 1 se puede observar que con la extracción de 3 componentes se tiene el $78,73 \%$ de la varianza total.

Tabla 1. Varianza total explicada por cada componente

\begin{tabular}{cccc}
\hline \multirow{2}{*}{ Componentes } & \multicolumn{3}{c}{ Autovalores iniciales } \\
\cline { 2 - 4 } & Total & \% de varianza & \% acumulado \\
\hline C1 & 34,487 & 48,625 & 48,625 \\
C2 & 14,855 & 20,945 & 69,57 \\
C3 & 6,519 & 9,192 & 78,763 \\
\hline
\end{tabular}

Para facilitar la interpretación del significado de los factores seleccionados se realizó una rotación de los ejes factoriales por el método de Varimax, desarrollado por Kaiser (1958), en este caso, y de acuerdo a la tabla 2 se observa que el componente 3 tiene la mayor varianza acumulada (46,8 \%) seguido del componente 2 que tiene una varianza de $21,6 \%$. 
Tabla 2. Componentes principales con rotación varimax

\begin{tabular}{cccc}
\hline \multirow{2}{*}{ Componentes } & \multicolumn{3}{c}{ Sumas de rotación de cargas al cuadrado } \\
\cline { 2 - 4 } & Total & \% de varianza & \% acumulado \\
\hline C1 & 7,255 & 10,23 & 10,23 \\
C2 & 15,389 & 21,698 & 31,928 \\
C3 & 33,216 & 46,834 & 78,763 \\
\hline
\end{tabular}

El factor F1 tiene correlación elevada con las variables: p37 y p16 (Área total de la finca), p17 (Área con bosque), p18 (Área con purma o barbecho), p21 (Área con pastos), p19 (Área con cultivo agroforestal), p20 (Área con cultivo en limpio), p41 (Movilidad y transporte), p36 (Título de propiedad de la finca) y p52 (Biomasa del SAF), las mismas que están vinculadas con los tipos de uso, área y propiedad de la finca. El factor 2 tiene correlación elevada con las variables: p55 (Tipo de SAF), p51 (Edad del SAF), p29 (Textura del suelo) y p8 (Número de personas que viven en la finca), las mismas que están vinculadas con las características del SAF. El factor 3 tiene correlación elevada con las variables: p9 (Localidad donde está la finca), p40 (Tipo de vivienda del agricultor) y p2 (Edad del agricultor), las mismas que están vinculadas con aspectos socio geográficos (tabla 3).

Tabla 3. Identificación e interpretación de los factores extraídos

\begin{tabular}{cccc}
\hline Factores & Variable & Carga factorial & Interpretación \\
\hline \multirow{5}{*}{ Primero } & p37 & 0,951 & \\
& p16 & 0,951 & \\
& p17 & 0,81 & \\
& p18 & 0,758 & \\
& p21 & 0,467 & Tipos de uso, área y \\
& p19 & 0,443 & propiedad de la finca \\
& p20 & 0,402 & \\
& p41 & 0,376 & \\
& p36 & 0,357 & \\
& p52 & 0,265 & \\
& p55 & 0,99 & \\
\hline \multirow{5}{*}{ Segundo } & p51 & 0,599 & \\
& p29 & 0,435 & Características del SAF \\
& p8 & 0,327 & \\
\hline \multirow{5}{*}{ Tercero } & P9 & 0,977 & \\
& P50 & 0,433 & \\
& P40 & 0,339 & Aspectos sociogeográficos \\
& P10 & 0,33 & \\
\hline
\end{tabular}




\section{Análisis de Clusters o Conglomerados}

Con las variables sintéticas generadas en el análisis de componentes principales se procedió al análisis cluster, con el propósito de formar grupos de sistemas agroforestales similares respecto de las tres nuevas variables creadas (sintéticas): Tipos de uso, área y propiedad de la finca, características del sistema agroforestal y aspectos socio geográficos. Se utilizó la medida de distancia también llamada medida de disimilitud y desemejanza, aplicándose la distancia de Manhattan, con el cual se tuvo 5 clusters o grupos de agricultores (figura 2).

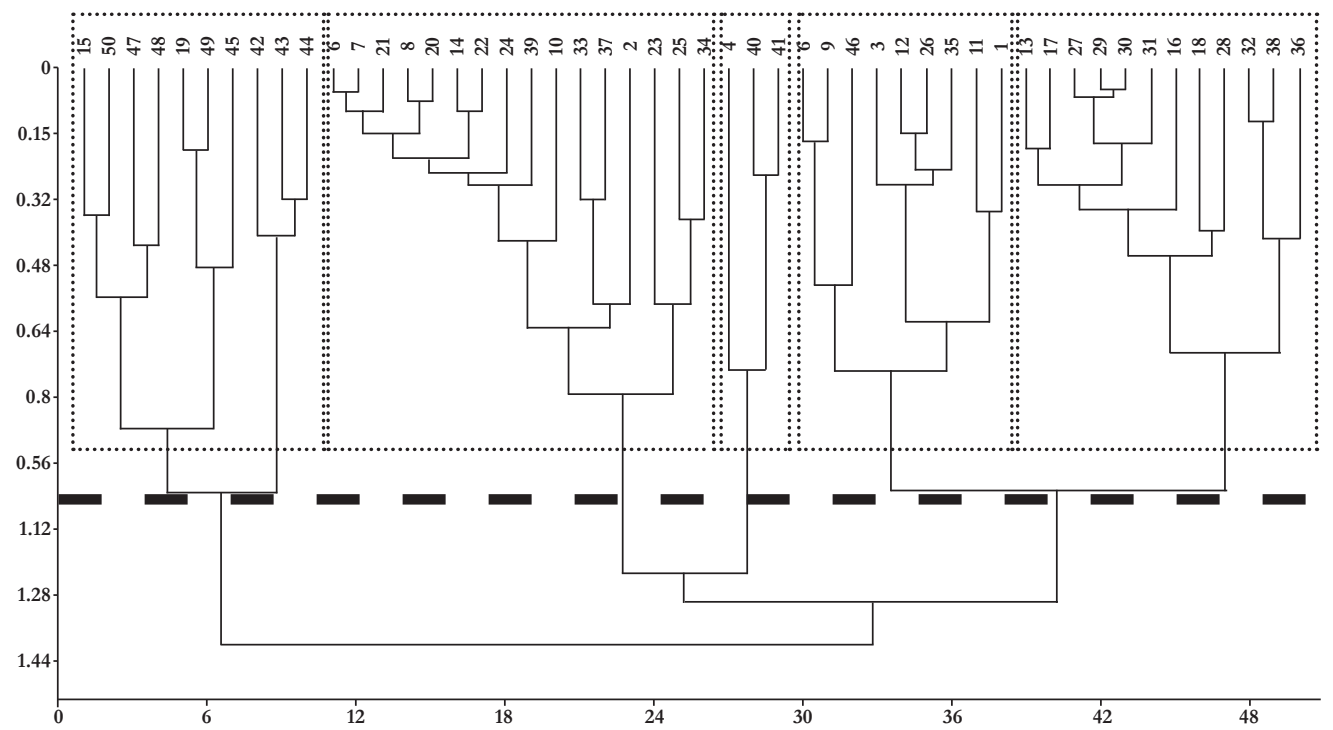

Figura 2. Análisis cluster y formación de grupos de SAFs.

\section{Tipos de Unidades de Producción Agroforestal}

En los SAFs evaluados se identificaron las siguientes especies cultivadas: cacao (Theobroma cacao), copoazu (Theobroma grandiflorum), naranja (Citrus sinensis), plátano (Musa paradisiaca), piña (Ananas comosus) y arazá (Eugenia stipitata) con la combinación de especies forestales como: achihua (Jacaranda copaia), pashaco (Schizolobium amazonicum), castaña (Bertholletia excelsa), guaba (Inga sp), sapote (Matisia cordata), tornillo (Cedrelinga catenaeformis), teca (Tectona grandis), caucho (Hevea brasiliensis), cetico (Cecropia sp), tahuari (Tabebuia serratifolia), pijuayo (Bactris gasipaes), aguaje (Mauritia flexuosa), huasai (Euterpe precatoria), capirona (Calicophyllum megistocaulum), shimbillo (Inga sp) y lupuna (Cavanillesia umbellata).

El Sistema Agroforestal tipo I está conformado por 10 agricultores (tabla 4), los mismos que tienen sus fincas en los distritos de Inambari, Tambopata, Laberinto y Las Piedras a base de las especies de cacao, copoazu, naranjas y plátanos con las especies 
forestales de achihua, pashaco, lupuna, castaña, guaba y sapote. El área total de la finca tiene un promedio de 30,5 ha, y el área destinada a sistemas agroforestales en promedio es de 5,0 ha. La edad promedio del SAF es 10 años, y la edad promedio del agricultor es 61 años, y tiene ingresos mensuales entre S/ 500-1700/mes (figuras 3 y 4).

Tabla 4. Ubicación de las parcelas del SAF tipo I

\begin{tabular}{cllll}
\hline Codigo & \multicolumn{1}{c}{ Agricultor } & \multicolumn{1}{c}{ Localidad } & \multicolumn{1}{c}{ Distrito } & \multicolumn{1}{c}{ Asociacion de especies } \\
\hline a15 & Filimon Leon Talaverano & Centromin & Inambari & copoazu-araza-achihua-pashaco-lupuna \\
a19 & Gladis Llicahua & Nueva Arequipa & Inambari & cacao-copoazu-guaba-pashaco \\
a42 & Enock Lazo & Chorrillos & Tambopata & copoazu-castaña-cedro-pashaco \\
a43 & Alfredo Vela Vera & Chorrillos & Tambopata & copoazu-naranja-guaba \\
a44 & Pedro Casanova Romero & Rompeolas & Tambopata & copoazu-chimicua-caoba-quillabordon \\
a45 & Lino Melgar Quispe & Piñal & Las Piedras & cacao-guaba-pashaco \\
a47 & Maria Champi Huaman & Florida Alta & Laberinto & copoazu-macambo-carambola-castaña \\
a48 & Elias Vela Moreno & Infierno & Tambopata & copoazu-platano-teca-sapote \\
a49 & Herbert Lobon & Bajo Tambopata & Tambopata & cacao-platano-ishpingo-tahuari \\
a50 & Victor Zambrano Gonzales & Bajo Tambopata & Tambopata & cacao-naranja-castaña-sapote-mamey \\
\hline
\end{tabular}

El Sistema Agroforestal tipo II (tabla 5) conformado por 16 agricultores que tienen sus fincas en los distritos de Inambari y Las Piedras a base de las especies de copoazu, piña, naranjas y plátanos con las especies forestales de castaña, tornillo, pashaco, teca, caucho, cetico , achihua, tahuari, pijuayo y aguaje. El área total de la finca tiene un promedio de 33,3 ha y el área destinada a sistemas agroforestales en promedio es de 8,8 ha (figuras 3 y 4). La edad promedio del SAF es 11,3 años, y la edad promedio del agricultor es 48,8 años, y tiene ingresos mensuales entre S/ 1500-2500/mes.

Tabla 5. Ubicación de las parcelas del SAF tipo II

\begin{tabular}{cllll}
\hline Codigo & \multicolumn{1}{c}{ Agricultor } & \multicolumn{1}{c}{ Localidad } & \multicolumn{1}{c}{ Distrito } & \multicolumn{1}{c}{ Asociacion de especies } \\
\hline a2 & Agustin Maqque Huamani & San Juan & Inambari & copoazu-piña-pijuayo-castaña \\
a6 & Julia Espinoza Baldez & El Progreso & Inambari & copoazu-pashaco-castaña-tornillo \\
a7 & Luis Huanca Quispe & El Progreso & Inambari & copoazu-aguaje-castaña-tornillo \\
a8 & Nicolas Huanca Quispe & El Progreso & Inambari & copoazu-castaña \\
a10 & Victor Huaman Quispe & Nueva esperanza & Inambari & cacao-copoazu-pashaco-sacsa-teca \\
a14 & Santos Ccuito Flores & El Progreso & Inambari & copoazu-achihua-castaña-tornillo \\
a20 & Donato Huanca Jaqquehua & El Progreso & Inambari & copoazu-castaña-leucaena \\
a21 & Sixto Huanca Jaqquehua & El Progreso & Inambari & copoazu-platano-achihua-castaña-pacay \\
a22 & Rufino Huanca Tocote & El Progreso & Inambari & copoazu-naranja-achihua-castaña-pijuayo \\
a23 & Sabino Huaman Ccopa & Sabaluyoc & Las Piedras & copoazu-platano-pashaco \\
a24 & Carlos Lopez Lopez & Planchon & Las Piedras & copoazu-platano-caucho-castaña \\
a25 & Luciano Quispe Chani & Alegria & Las Piedras & copoazu-platano-caucho \\
a33 & Gotardo Roque Guerra & Alegria & Las Piedras & copoazu-naranja-castaña-pashaco \\
a34 & Rosa Mamani Molina & Alegria & Las Piedras & copoazu-platano-teca-castaña \\
a37 & Charles Tello Papa & Alegria & Las Piedras & limon-pasahaco-tahuari \\
a39 & Aderly Ccuito Flores & El Progreso & Inambari & piña-cetico-tornillo \\
\hline
\end{tabular}


El Sistema Agroforestal tipo III está conformado por un agricultor y dos instituciones de investigación existentes en Madre de Dios (tabla 6), se trata del Instituto de Investigaciones de la Amazonia Peruana (IIAP) y del Instituto Nacional de Investigación e Innovación Agraria (INIA), en cuyos campos experimentales existen sistemas agroforestales a base de las especies de piña, copoazu, plátano, y arazá con las especies forestales de castaña, achihua, tornillo, huasai y pijuayo. El área total de la finca tiene un promedio de 116 ha y el área destinada a sistemas agroforestales en promedio es de 13,6 ha (figuras 3 y 4). La edad promedio del SAF es 12,3 años, y la edad promedio del agricultor es 46,6 años, y tiene ingresos mensuales entre S/ 2000-3500/mes.

Tabla 6. Ubicación de las parcelas del SAF tipo III

\begin{tabular}{cllll}
\hline Codigo & \multicolumn{1}{c}{ Agricultor } & \multicolumn{1}{c}{ Localidad } & \multicolumn{1}{c}{ Distrito } & \multicolumn{1}{c}{ Asociacion de especies } \\
\hline a4 & Cirilo Mendez Villafane & San Juan & Inambari & piña-achihua-castaña-tornillo \\
a40 & IIAP & Fitzcarrald & Tambopata & copoazu-platano-huasai-castaña \\
a41 & INIA & San Bernardo & Tambopata & copoazu-araza-castaña-pijuayo \\
\hline
\end{tabular}

El Sistema Agroforestal tipo IV está conformado por 9 agricultores (tabla 7), los mismos que tienen sus fincas en los distritos de Inambari y Las Piedras a base de las especies de cacao, copoazu y naranja con las especies forestales de guaba, pashaco, tornillo, castaña, capirona y pijuayo. El área total de la finca tiene un promedio de 69,4 ha y el área destinada a sistemas agroforestales en promedio es de 7,6 ha (figuras 3 y 4). La edad promedio del SAF es 5,3 años, y la edad promedio del agricultor es 53 años, y tiene ingresos mensuales entre S/ 1000-2500/mes.

El Sistema Agroforestal tipo V conformado por 12 agricultores (tabla 8), los mismos que tienen sus fincas en los distritos de Inambari y Las Piedras a base de las especies de cacao, naranja y plátano con las especies forestales de guaba, pashaco, achihua, tornillo, castaña, shimbillo, lupuna y pijuayo. El área total de la finca tiene un promedio de 23,7 ha y el área destinada a sistemas agroforestales en promedio es de 5,7 ha (figuras 3 y 4). La edad promedio del SAF es 7,5 años, y la edad promedio del agricultor es 47,8 años, y tiene ingresos mensuales entre S/ 500-1500/mes.

Tabla 7. Ubicación de las parcelas del SAF tipo IV

\begin{tabular}{cllll}
\hline Codigo & \multicolumn{1}{c}{ Agricultor } & \multicolumn{1}{c}{ Localidad } & \multicolumn{1}{c}{ Distrito } & \multicolumn{1}{c}{ Asociacion de especies } \\
\hline a1 & David Huaman Ramos & El Progreso & Inambari & cacao-guaba-castaña \\
a3 & Soledad Apaza Mamani & San Juan & Inambari & cacao-copoazu-platano-guaba \\
a5 & Agueda Calisaya Cervantes & San Juan & Inambari & cacao-guaba \\
a9 & Alejandrina Mamani Quispe & Santa Rosa & Inambari & cacao-aguaje-pashaco-tornillo \\
a11 & Faustino urbano Batallanos & Nueva esperanza & Inambari & cacao-capirona-cast-lupuna-pijuayo-torn. \\
a12 & Efrain Pizarro Cordova & Santa Rosa & Inambari & cacao-naranja-guaba \\
a26 & Carmelo Carmen Hancco & Alegria & Las Piedras & cacao-naranja-guaba-castaña \\
a35 & Victor Vargas Guerra & Alegria & Las Piedras & cacao-guaba-achihua-pashaco \\
a46 & Morgan Gonzales Chavez & San Juan & Inambari & cacao-copoazu-guaba \\
\hline
\end{tabular}


Tabla 8. Ubicación de las parcelas del SAF tipo V

\begin{tabular}{lllll}
\hline Código & \multicolumn{1}{c}{ Agricultor } & \multicolumn{1}{c}{ Localidad } & \multicolumn{1}{c}{ Distrito } & \multicolumn{1}{c}{ Asociación de especies } \\
\hline a13 & Andres Parisaca & Santa Rosa & Inambari & cacao-naranja-guaba \\
a16 & Ricardo Torres Fernandez & Puerto Trujillo & Inambari & cacao-plátano-pijuayo-guaba-pashaco \\
a17 & Hermenegildo Conza Huallpa & Villa Sntiago & Inambari & cacao-plátano-pacay-pashaco \\
a18 & Teodoro Mamani Yanque & Villa Sntiago & Inambari & cacao-castaña-cedro-lupuna-pijuayo \\
a27 & Yoni Curinambe Leiva & San Francisco & Las Piedras & cacao-guaba-achiua \\
a28 & Jhon Ayala Chacon & San Francisco & Las Piedras & cacao-guaba \\
a29 & Henrry Curinambe Leiva & San Francisco & Las Piedras & cacao-tornillo-achiua \\
a30 & Fredy Curinambe Leiva & San Francisco & Las Piedras & cacao-guaba-tornillo \\
a31 & Estanislao Curinambe Chavez & San Francisco & Las Piedras & cacao-guaba \\
a32 & Basilides Mamani Puma & Alegría & Las Piedras & cacao-naranja-castaña \\
a36 & Ramon Tineo Sandoval & Alegría & Las Piedras & cacao-plátano-guaba \\
a38 & Tito Tuesta Ortiz & Alegría & Las Piedras & cacao-plátano-shimbillo-lupuna \\
\hline
\end{tabular}

\section{DISCUSIÓN}

Con la finalidad de reducir el número de variables en el análisis multivariado, en un primer momento solo se consideraron a las variables cuyo coeficiente de variabilidad fuera superior a $50 \%$, y es ahí donde se reduce el número de variables de 64 a 15 . Para continuar con la reducción se hizo el Análisis de Componentes Principales a las 15 variables resultantes, y en esta primera corrida (tabla 1), los tres primeros componentes logran explicar el 78,76\% de la varianza total, valor que está entre 70-80\% que son puntos de corte óptimo planteados por Manly (1986) y Afifi y Clark, (1999); en la segunda corrida donde se hizo la rotación varimax se observa que los porcentajes de la varianzas de los tres componentes antes mencionados se invierten, de tal manera que el componente 3 tiene el mayor porcentaje de varianza, y el componente 1 tiene el menor porcentaje (tabla 2). Habiéndose reducido las variables a tres factores y generándose tres variables sintéticas, el análisis cluster nos permite identificar cinco tipos de Sistemas Agroforestales.

Las variables vinculadas al tipo de uso, área y propiedad de la finca, las características del SAF y los aspectos socio geográficos fueron importantes para poder hacer la tipificación en el presente estudio, resultados que son similares a los encontrados por Hernández et al. (2013), quienes sugieren que variables como tamaño de la finca, número de individuos, producción, ingresos y nivel educativo son las más útiles para clasificar a los productores. Köbrich et al. (2003) señalan que las diferencias en el tamaño de unidad de producción, tenencia de la tierra, tipo de tierras y nivel de mecanización son factores reconocidos en la formación de conglomerados. Así mismo; Solano et al. (2001) y Gaspar et al. (2011) reportan hallazgos similares. Por otro lado, Tuesta et al. (2014) en la tipificación de Sistemas Agroforestales con cacao encontraron que las variables más importantes son Tecnología y Gestión, Económico, Familiar, Actividad Agrícola y Recursos Naturales. 
El área del SAF tipo III es mayor que en el resto de los SAF, mientras que los SAF tipo I y V tienen sus áreas más pequeñas (figuras 3a y 3b). La combinación de especies forestales y cultivadas en los SAF IV y V es casi similar (figura 3c). Por otro lado, los SAF tipo IV y V son los más jóvenes que tienen entre 4 y 7 años de instalados (figura 4a). Los mayores ingresos económicos mensuales se observan en el SAF III, mientras que en los SAF I y V se tienen los ingresos mensuales más bajos (figura 4b).
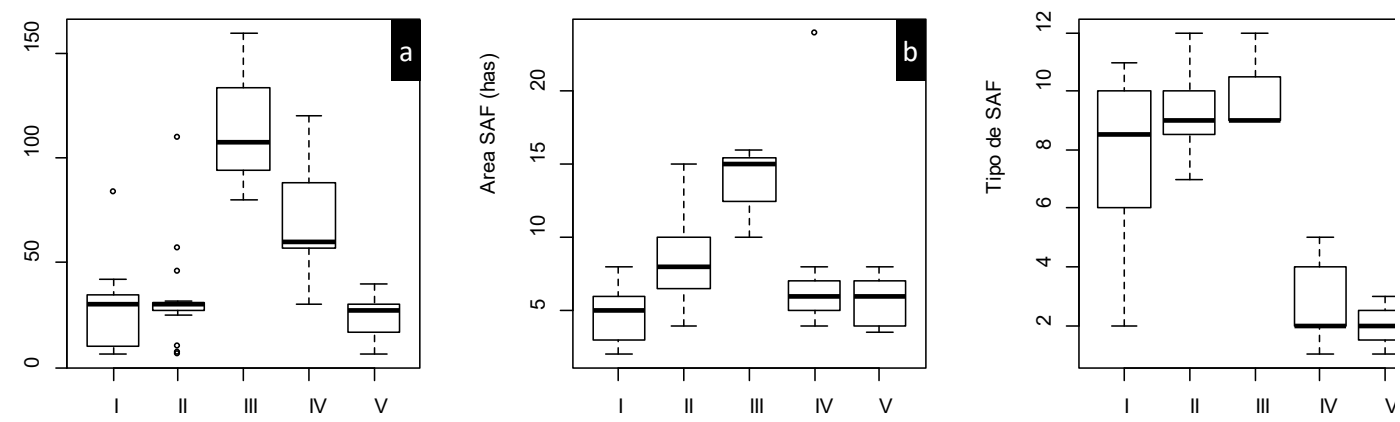

Figura 3. Características de la finca: a) Área total, b) Área del SAF y c) Tipo de SAF
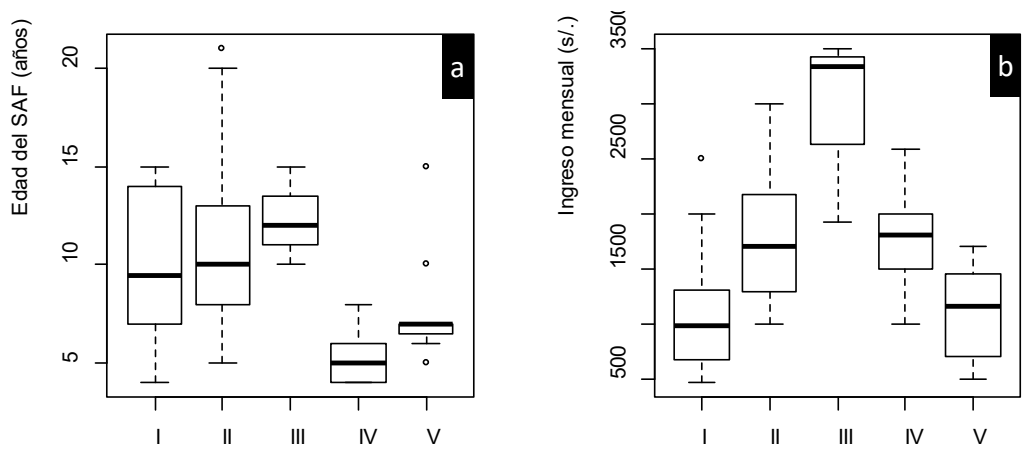

Figura 4. Características de la finca: a) Edad del SAF y b) Ingreso mensual/agricultor.

\section{CONCLUSIONES}

Con la metodología de análisis de datos planteada se logró identificar 5 tipos de sistemas agroforestales en la Provincia de Tambopata, y las variables sintéticas que fueron útiles para la tipificación fueron las que están asociadas con: a) el tipo de uso, área y propiedad de la finca, b) las características del SAF. y c) los aspectos socio geográficos.

El SAF tipo I tiene una edad promedio de 10 años y 5 ha. destinadas a la agroforesteria, con cultivos principales de copoazu y cacao asociado hasta con 4 especies forestales. El SAF tipo II tiene una edad promedio de 11,3 años y 8,8 ha. destinadas a la agroforesteria, con cultivo de copoazu asociado hasta con 5 especies forestales. El SAF tipo III tie- 
ne una edad promedio de 12,3 años y 13,6 ha destinadas a la agroforesteria, con cultivo de copoazu y piña asociado hasta con 3 especies forestales. Los SAF tipo IV y V tienen una edad promedio de 5,3 y 7,5 años respectivamente con menos de 7,6 ha destinadas a la agroforesteria, con cultivo de cacao joven asociada a 2 especies forestales.

\section{REFERENCIAS BIBLIOGRÁFICAS}

Afifi, Abdelmonem y Clark, Virginia. 1999. Computer-Aided multivariate analysis. $3^{\circ}$ edición. Texts in Statistical Science. Washington D.C. Chapman y Hall CRC. Boca Raton.

Escobar, Germán y Berdegué, Julio. 1990. Tipificación de sistemas de producción agrícola. Red Internacional de Metodología de Investigacion de Sistemas de Producción RIMISP. Santiago de Chile.

Gaspar, P. y otros. 2011. «Goat systems of Villuercas-Ibores area in SW Spain: Problems and perspectives of traditional farming systems». Small Ruminant Research. Vol 97: 1-11.

Hernández, Patricia y otros. 2013. «Tipificación de los sistemas campesinos de producción de leche del sur del estado de México». Universidady ciencia, Vol. 29, N 1. México: Universidad Juárez Autónoma de Tabasco: 19-31. <http:/ / www.scielo. org.mx/scielo.php?script=sci_arttext\&pid=S0186-29792013000100003\&lng=e s\&tlng=es $>$ [Consulta: 10-06-2017].

Kaiser, Henry. (1958). «The varimax criterion for analytic rotation in factor analysis». Psychometrika, Vol. 23, No 3: 187-200.

Köbrich, C; Rehman, T. y Khan, M. 2003. Typification of farming systems for constructing representative farm models: two illustrations of the application of multivariate analyses in Chile and Pakistan. Agricultural Systems 76:141-157.

Manly, Bryan. 1986. Multivariate Statistical Methods. Springer; 1 ra edi. Nueva Zelanda, 159.

Por la tierra (2015). Asociación de Productores El Progreso: Yanaocas en los Andes y la Amazonía. Estudio de caso. Movimiento regional por la tierra - Peru. < http:// www.porlatierra.org/casos/ver/49> [Consulta: 10-07-2016].

Solano, Carolina y otros. 2001. Who makes farming decisions? A study of Costa Rica dairy farmers. Agricultural Systems 67: 181-199. 
Tuesta, Oscar y otros. 2014. «Tipología de fincas cacaoteras en la subcuenca media del río Huayabamba, Distrito de Huicungo (San Martín, Perú) ». Ecología Aplicada, 13(2). http://www.lamolina.edu.pe/ecolapl/Articulo\%208\%20No \%202\%20 vol\%2013.pdf [Consulta: 13-06-2017].

Recibido: $18 / 07 / 17$

Aceptado: 26/10/17

\section{Correspondencia}

Joel Peña Valdeiglesias

nuevojoel@yahoo.es 